\title{
Mixing Time and Liquid Circulation Rate in Steelmaking Ladles with Vertical Gas Injection
}

\author{
Hasmet TURKOGLU and Bakhtier FAROUK \\ Mechanical Engineering and Mechanics Department, Drexel University, Philadelphia, PA 19104, U.S.A.
}

(Received on April 10, 1991; accepted in final form on September 20, 1991)

\begin{abstract}
Flow and concentration fields in bench scale gas-injected molten steel baths were numerically studied to investigate dependency of flow structure and mixing characteristics on bath aspect ratio and gas injection rate. The problem was formulated as a two-phase flow on the basis of Eulerian approach for both gas and liquid phase transports. The effects of vessel aspect ratio and gas injection rate on mixing efficiency were characterized from the predicted time evolution of a tracer distribution in the melt and also from the liquid circulation rate. Results demonstrated that both vessel aspect ratio and gas injection rate play crucial roles on flow structure in the bath and hence on the mixing efficiency. It was observed that there is an optimum vessel aspect ratio $(\sim 1.5)$ for minimum mixing time for the systems considered. There also exists an optimum gas injection rate for maximum mixing intensity (minimum mixing time) in a given system.
\end{abstract}

KEY WORDS: ladle metallurgy; mixing power; mixing time.

\section{Introduction}

In the present study, mixing characteristics in argon stirred molten steel baths were studied. Vertical injection of argon into a molten steel bath contained in a cylindrical vessel was considered. The problem was formulated on the basis of a multi-component multi-phase model using the Eulerian approach for both phases. Fluctuating motion of the liquid free surface and its irregular shape were taken into account by extending the computational domain above the initial undisturbed liquid height. The turbulence in the liquid phase was computed using the two-equation $(k-\varepsilon)$ model. The flow and volume fraction fields in both the gas and liquid phases were predicted numerically. To obtain the mixing time, a tracer liquid was initially injected from top of the vessel, and time evolution of the tracer concentration distribution in the bath was solved. The liquid circulation rate and mixing time were used as measures for intensity of mixing in the bath. 'Mixing time', $\tau_{m}$, is defined as the time taken for the tracer concentration to attain, at every nodal location in the system, a value within $\pm 5 \%$ of the tracer concentration of the well mixed steady state bath. 'The liquid circulation rate' is defined here as the total amount of liquid flowing upward (or downward) through the horizontal mid-plane. In the tracer concentration equation, both the convection and diffusion processes were accounted for. The effects of vessel aspect ratio $\left(H_{i} / D\right)$ and gas injection rates on the mixing time, the liquid circulation rate and on the flow structure in general were investigated.

\section{Background}

A considerable body of theoretical and experimental work concerning gas injection systems has been undertaken in recent years. In most of these studies, air and water were used to model gas injection in metallurgical processes. ${ }^{1-12)}$ In these studies it was assumed that gas penetration is confined in a cylindrical or conical region around the axis of the injection nozzle. Single phase flow equations were solved for the liquid phase with spatially varying density. The density of the mixture was calculated as a function of the phase volume fractions. Boundaries of the gas-liquid two-phase plume were determined from experimental observations. To investigate the structure of the two-phase plume region, Castillejos and Brimacombe ${ }^{13-15)}$ performed experimental studies in air-water, nitrogen-mercury and helium-mercury systems. From the results of these studies they suggested correlations for the gas phase volume fraction distribution as a function of fluid properties and system geometry.

Realizing the limitations of the single phase formulation, McKelliget et al..${ }^{16)}$ Lai and Salcudean, ${ }^{17)}$ Salcudean and $\mathrm{Lai}^{1{ }^{8)}}$ and Lai et al. ${ }^{19)}$ used a mathematical model in which Navier-Stokes equations were solved for the liquid phase, while a set of simplified conservation equations were solved to predict the gas phase flow. Aldham et al. ${ }^{20)}$ Cross and Markatos, ${ }^{21,22)}$ Koh et al. ${ }^{23)}$ and Turkoglu and Farouk ${ }^{24-26)}$ used a two-phase flow model to predict the flow fields in the gas and liquid phases. Two sets of governing equations were solved for gas and liquid phases. Johansen and Boysan ${ }^{27)}$ used a two-phase flow model based on the Eulerian-Lagrangian 
approach. Two-phase flow formulation used in above studies does not require any experimental information or assumption on gas plume boundary. However, the experimental information on the bubble sizes and on the inter-phase exchange of momentum is needed.

Mixing characteristics of gas injection systems have mostly been studied experimentally by injection of a tracer into a liquid bath. The concentration of the tracer was detected at one or more points in the vessel, and the mixing time was correlated against the specific mixing power, $\dot{\varepsilon}_{m}$. Specific mixing power is the rate of work done on per unit mass of the liquid phase by the injected gas. The first correlation was given by Nakanishi et al. ${ }^{28)}$ They obtained mixing time data in a water model of argon stirred molten steel, in an induction stirred system and also in a vacuum agitated unit, and proposed the following expression for the mixing time:

$$
\tau_{m}=800 \dot{\varepsilon}_{m}^{-0.4}
$$

where $\dot{\varepsilon}_{m}$ (in $\mathrm{W} /$ ton) is the mixing power which was calculated for argon-stirred ladles assuming that gas injection pressure was the same as atmospheric pressure, and that gas temperature reaches the liquid temperature at the nozzle exit. These authors claimed that mixing is mainly controlled by eddy viscosity rather than bulk circulation of the fluid in the vessel. Similar expressions with different exponents and coefficients were also suggested by other investigators. ${ }^{29)}$ The calculation of mixing power differs slightly in those correlations. In a later study, Szekely et al. $^{30)}$ analyzed the flow in vertical gas injection systems through numerical solution of governing equations of the fluid motion and the mass concentration equation for a tracer fluid. They performed computations in pilot scale and industrial size argon stirred ladles. The prediction of mixing times was found to be in qualitative agreement with experimentally measured values. Haida et al. $^{31)}$ reported mixing time measurements in a water model of an argon-stirred ladle. These authors noted that mixing times measured in the water model ladle were considerably different from those obtained on the basis of the correlation proposed by Nakanishi et al. ${ }^{28)}$

Asai et al. ${ }^{32)}$ investigated the role of eddy diffusion and bulk convection on mixing phenomena. On the basis of measurements, they concluded that mixing time does not depend on vessel size in the viscous regime (low gas injection rate), but does depend on vessel size in inertia dominated systems (high gas injection rate). On the basis of their analytical study, Sano and Mori ${ }^{33)}$ argued that mixing time is strongly affected by the dimensions of the liquid bath. Oymo and Guthrie ${ }^{34)}$ experimentally investigated the mixing time in a combination blowing water model of gas stirred steel bath. They found that location of the bubblers is an important factor in mixing time. Similar conclusions were obtained by Matway et $a .^{35)}$ on the basis of their experimental measurements. A recent study carried out by Joo et $a l^{36)}$ also showed the importance of tuyere position on mixing time. Mazumdar and Guthrie ${ }^{37)}$ investigated the dependency of the mixing time on the diffusion, bulk convection, and on the bath dimensions. Their work, which also contains a good critical review of previous studies on the mixing time, was based on experimental and analytical studies. The authors concluded that both eddy diffusivity and bulk convection play important roles on the mixing in the vessel together with bath dimensions. They proposed a semiempirical correlation for the mixing time as a function of the mixing power and bath dimensions (radius and liquid height).

$$
\tau_{m}=37 \dot{\varepsilon}_{m}^{-1 / 3}(D / 2)^{5 / 3} H_{i}^{-1}
$$

The mixing power $\dot{\varepsilon}_{m}$ (in $\mathrm{W} / \mathrm{kg}$ ) was calculated as

$$
\dot{\varepsilon}_{m}=\frac{\rho_{L} g Q H_{i}}{\rho_{L} \pi(D / 2)^{2} H_{i}}
$$

In this calculation, the contribution of the gas kinetic energy at the nozzle exit to the mixing power was neglected entirely and gas flow rate was corrected to the mean height and temperature of the liquid in vessel. For the derivation of Eq. (2), the authors assumed that the average liquid circulation velocity in the liquid region away from the axis of the injection nozzle was uniform. They also assumed that the eddy kinematic viscosity has a uniform value over the entire bath. These parameters were predetermined using ad hoc relations, suggested by Sahai and Guthrie. ${ }^{7)}$ The constant coefficient in Eq. (2) was determined using the mixing time data obtained in air-water systems, in which water was agitated by air injection through a central vertical lance.

In the present study, the mixing characteristics in gas injected molten metal baths are numerically studied. The effects of vessel aspect ratio $\left(H_{i} / D\right)$ and gas injection rate on the flow structure and on the mixing efficiency are investigated. Results are obtained for bench scale steelmaking ladles with argon injection. Fully time dependent calculations are carried out for the prediction of the two-phase flow characteristics within the ladle. The mixing efficiency is quantified by mixing time and the liquid circulation rate.

\section{Mathematical Formulation}

The mathematical model used in the present study was based on a two-fluid model. ${ }^{38)}$ Gas and liquid phases were considered to be two different continuous fields interpenetrating and interacting with each other. Mass conservation, momentum and energy equations were derived for both phases considering time averaged variables. It was assmed that phases exchange momentum through finite interfacial surface area. Pressure in both phases was assumed to be the same within a small control volume. Field equations for each phase were weighed with volume fraction of that phase. In the conservation equations, exchanges between the phases were represented by source/sink terms. Phase dispersion due to turbulence was formulated by introducing a diffusion term in the mass conservation equation of the gas phase. Phase dispersion coefficient was calculated from turbulent viscosity of the continuous phase by introducing a dispersion Prandtl number. 


\section{Governing Equations}

\subsection{Mass Conservation Equation}

In the absence of phase change, the mass balance for phase ' $i$ ' with volume fraction $R_{i}$ yields to

$$
\frac{\partial}{\partial t}\left(R_{i} \rho_{i}\right)+\operatorname{div}\left(R_{i} \rho_{i} \vec{V}_{i}\right)-\operatorname{div}\left(\rho_{i} D_{i} \operatorname{grad} R_{i}\right)=0 \ldots
$$

where $\rho_{i}, \vec{V}_{i}$, and $D_{t}$, are phase density, velocity and turbulent diffusivity, respectively. In the above equation, the last term represents phase dispersion due to turbulent fluctuations. The turbulent phase diffusivity, $D_{t}$ was evaluated from the liquid phase turbulent viscosity by introducing a turbulent dispersion Prandtl number $\sigma_{d}$ as

$$
\sigma_{d}=\frac{v_{t}}{D_{t}}
$$

where $v_{t}$ is turbulent kinematic viscosity of the liquid phase, which was predicted using the two-equation $k-\varepsilon$ turbulence model. $\sigma_{d}$ was taken to be 0.1 using the results of our previous computations in air-water systems. ${ }^{24)}$ A similar approach was also used by Mostafa and Mongia $^{39)}$ for calculations of droplet dispersion in sprays.

\subsection{Momentum Equation}

Let $u_{i l}$ be velocity of phase ' $i$ ' in direction ' $l$ '. Then the momentum equation for phase ' $i$ ' in $l$-direction can be written as

$$
\begin{aligned}
\frac{\partial}{\partial t}\left(R_{i} \rho_{i} u_{i l}\right) & +\operatorname{div}\left(R_{i} \rho_{i} \vec{V}_{i} u_{i l}\right)=-R_{i} \vec{I} \cdot \operatorname{grad} p+B_{i l} \\
& +\operatorname{div}\left(R_{i} \mu_{\mathrm{eff}, i} \operatorname{grad} u_{i l}\right)+I_{i l} \ldots \ldots \ldots \ldots \ldots \ldots
\end{aligned}
$$

where $B_{i l}$ is body force in $l$-direction acting on phase ' $i$ ' per unit volume. $\mu_{\text {eff }}\left(\mu_{\text {eff }, i}=\mu_{i}+\mu_{t, i}\right)$ is the effective viscosity of the phase ' $i$ '. $I_{i l}$ is the momentum transfer to the phase ' $i$ ', in $l$-direction, resulting from interactions with the other phase in the control volume.

\subsection{Tracer Concentration Equation}

The tracer was considered as a second component in the liquid phase (having the same thermophysical properties of the molten steel). The tracer concentration $C$ was calculated considering only mass of tracer and melt in a small control volume. Then, the tracer concentration (tracer mass fraction) equation is written as

$$
\frac{\partial}{\partial t}\left(R_{L} \rho_{L} C\right)+\operatorname{div}\left(\vec{V}_{L} R_{L} \rho_{L} C\right)=\operatorname{div}\left(R_{L} \Gamma_{\text {eff }, C} \operatorname{grad} C\right)
$$

where $\Gamma_{\text {eff,c }}$ is the effective mass diffusion coefficient, which is defined as

$$
\Gamma_{\text {eff, } C}=\frac{\mu_{L}}{S c}+\frac{\mu_{t, L}}{S c_{t}}
$$

$S c$ and $S c_{t}$ are laminar and turbulent Schmidt numbers, respectively. Both were taken to be unity in this study.

\subsection{Turbulent Kinetic Energy Equation for Liquid Phase}

$\frac{\partial}{\partial t}\left(R_{L} \rho_{L} k\right)+\operatorname{div}\left(R_{L} \rho_{L} \vec{V}_{L} k\right)=\operatorname{div}\left(R_{L} \frac{\mu_{\mathrm{eff}, L}}{\sigma_{k}} \operatorname{grad} k\right)+S_{k}$

where,

$$
\begin{array}{r}
S_{k}=R_{L}\left(G_{k}-\rho_{L} \varepsilon\right) \ldots \ldots \ldots \ldots \ldots \ldots \ldots \ldots \ldots \ldots \ldots \ldots \ldots \ldots \ldots \\
G_{k}=\mu_{t, L}\left\{2\left[\left(\frac{\partial v_{L}}{\partial r}\right)^{2}+\left(\frac{\partial w_{L}}{\partial z}\right)^{2}+\left(\frac{v_{L}}{r}\right)^{2}\right]\right. \\
\left.+\left(\frac{\partial w_{L}}{\partial r}+\frac{\partial v_{L}}{\partial z}\right)^{2}\right\} \ldots \ldots \ldots \ldots \ldots \ldots \ldots \ldots \ldots
\end{array}
$$

$\sigma_{k}$ is a turbulence model constant, and $S_{k}$ is source of the turbulent kinetic energy.

\subsection{Equation of Dissipation Rate of Turbulent Kinetic Energy for Liquid Phase}

$\frac{\partial}{\partial t}\left(R_{L} \rho_{L} \varepsilon\right)+\operatorname{div}\left(R_{L} \rho_{L} \vec{V}_{L} \varepsilon\right)=\operatorname{div}\left(R_{L} \frac{\mu_{\mathrm{eff}, L}}{\sigma_{\varepsilon}} \operatorname{grad} \varepsilon\right)+S_{\varepsilon}$

where $S_{\varepsilon}$ is the source term.

$$
S_{\varepsilon}=R_{L} \frac{\varepsilon}{k}\left(C_{1} G_{k}-C_{2} \rho_{L} \varepsilon\right)
$$

Model constants used in this study were the same as those used in single phase flows. These are given in Table 1. The turbulence viscosity is calculated as

$$
\mu_{t, L}=C_{\mu} \frac{\rho_{L} k^{2}}{\varepsilon}
$$

\subsection{Interphase Momentum Exchange}

Momentum exchange between the phases can be expressed in terms of forces acting between the phases. For calculation of inter-phase force, information regarding bubble/droplet size, local phase volume fractions, local relative velocity and the drag coefficient of bubble or droplet is required. In accordance with the phase momentum equations given above, momentum exchange between the phases can be written per unit volume as:

$$
I_{i l}=C_{f}\left(u_{j l}-u_{i l}\right)
$$

where $C_{f}$ is inter-phase friction coefficient. By neglecting the higher order transient effects, the inter-phase force per unit volume in the $l$-direction can also be written as ${ }^{40}$ )

$$
I_{i l}=\frac{C_{D} \frac{1}{2}\left|\vec{V}_{r}\right| A_{d} R_{d} \rho_{c}}{B_{d}}\left(u_{j l}-u_{i l}\right)
$$

where $\vec{V}_{r}$ is relative velocity, $A_{d}$ is the projected area of

Table 1. $k-\varepsilon$ turbulence model constants.

\begin{tabular}{ccccc}
\hline$C_{1}$ & $C_{2}$ & $C_{\mu}$ & $\sigma_{k}$ & $\sigma_{\varepsilon}$ \\
\hline 1.44 & 1.92 & 0.09 & 1.0 & 1.3 \\
\hline
\end{tabular}


a typical bubble or a droplet, $B_{d}$ is volume of a typical bubble or a droplet, $R_{d}$ is the volume fraction of the dispersed phase, and $C_{D}$ is the dimensionless drag coefficient. Here, subscripts ' $c$ ' and ' $d$ ' are used to denote continuous and dispersed phases, respectively. Comparing Eqs. (15) and (16), $C_{f}$ can be expressed as:

$$
C_{f}=\frac{C_{D} \frac{1}{2}\left|\vec{V}_{r}\right| A_{d} R_{d} \rho_{c}}{B_{d}}=\frac{3}{4} \frac{C_{D}\left|\vec{V}_{r}\right| R_{d} \rho_{c}}{d_{e}} \ldots . .
$$

$\vec{V}_{r}$ and $R_{d}$ are obtained from the solution of field equations. The drag coefficient $C_{D}$ and characteristic bubble/droplet diameter, $d_{e}$, are empirically or semiempirically determined parameters, which differs from one flow regime to another.

In gas injection systems the phase concentrations may change considerably over the flow domain, and in turn the flow regime changes. ${ }^{40)}$ In general, a highly concentrated gas region about the axis of the injection nozzle and a relatively lower gas concentration region away from the injection axis are observed. The highly concentrated gas region may be considered as a churn-turbulent flow, while the region away from the axis can be classified as a bubbly flow. In churn-turbulent regime a particle (bubble) can influence both the surrounding fluid and other particles directly. Formally, a 0.3 gas volume fraction can be used as a criteria to distinguish between these two flow regimes as suggested by Ishii and Zuber., ${ }^{40)}$

In the churn-turbulent flow regime, the drag coefficient can be calculated as $^{43)}$

$$
C_{D}=\frac{8}{3}\left(1-R_{d}\right)^{2}
$$

where $R_{d}$ is volume fraction of the dispersed phase. In the bubbly flow and droplet-gas regions, $C_{D}$ is calculated $\operatorname{as}^{41)}$

$$
C_{D}=\frac{24\left(1+0.15 \operatorname{Re}_{d}^{0.687}\right)}{\operatorname{Re}_{d}}+\frac{0.42}{1+\frac{4.25 \times 10^{4}}{\operatorname{Re}_{d}^{1.16}}}
$$

where $\operatorname{Re}_{d}$ is Reynolds number based on the bubble/droplet diameter and slip velocity.

\subsection{Initial and Boundary Conditions}

In practical gas injection systems, the location and shape of the liquid free surface does not remain flat and smooth as the erupting gas column breaks up the liquid surface. Soon after the gas jet is turned on, the "free surface' region becomes a two-phase zone characterized by high gradients of the liquid/gas volume fraction. This 'free surface' region is a natural outcome of the solution (flow structure in the bath) to the problem. In the present study, the computational domain is extended beyond the initial height of the undisturbed liquid level (see Fig. 1). The region near the free surface was considered to be within the computational domain and nodal points in that region were treated as regular internal grid points. An advantage of the present formulation is that reasonable estimates of the location and shape of the

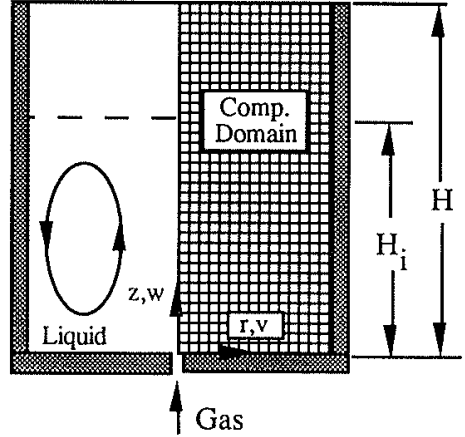

Fig. 1. Computational domain and coordinate system of the problem.

'free surface' region can be obtained without specifying additional conditions at this region. Assuming the flow is axisymmetric, the following initial and boundary conditions were used in the present analysis (see Fig. 1).

Initially, the bath was considered to be partially filled with quiescent liquid up to a height $H_{i}$ (as shown in Fig. 1). The space above the liquid was occupied by the gas only. Specifically:

$$
\begin{aligned}
& R_{G} \approx 0 \text { for } z \leq H_{i} \text { and } R_{G} \approx 1 \text { for } z>H_{i} \\
& R_{L} \approx 1 \text { for } z \leq H_{i} \text { and } R_{L} \approx 0 \text { for } z>H_{i}
\end{aligned}
$$

\subsection{Boundary Conditions}

(1) Along the axis: Symmetry conditions apply, i.e.,

$$
\begin{gathered}
v_{G}=v_{L}=0 \\
\frac{\partial w_{G}}{\partial r}=\frac{\partial w_{L}}{\partial r}=\frac{\partial k}{\partial r}=\frac{\partial \varepsilon}{\partial r}=0
\end{gathered}
$$

and

$$
\frac{\partial R_{G}}{\partial r}=\frac{\partial R_{L}}{\partial r}=0
$$

(2) Along the walls: The velocities satisfy the no slip condition, i.e.,

$$
v_{G}=v_{L}=w_{G}=w_{L}=0
$$

The values of $k$ and $\varepsilon$ near wall points, and friction on the walls were calculated using the logarithmic law of the wall. ${ }^{42)}$

(3) At the nozzle exit: Flat velocity profiles were assumed for the gas at the orifice exit. The gas phase turbulence was not modeled explicitly, as it was assumed that the effect of turbulent fluctuations in the gas jet was insignificant on the flow in the liquid. A zero gradient boundary condition was assigned for the liquid phase turbulence parameters $k$ and $\varepsilon$. Also note that the liquid volume fraction was considered to be negligible at nozzle exit.

(4) Along the top surface ( $z=H$ in Fig. 1): At the top surface (which is well above the initial undisturbed liquid height $z=H_{i}$ ), the gas was allowed to leave the system at the rate it arrives at the free surface. The pressure was set to atmospheric. The liquid was not allowed to leave the system by fixing the vertical velocity component to zero. The zero shear condition was applied for both phases, i.e., 


$$
\begin{aligned}
& \frac{\partial v_{G}}{\partial z}=\frac{\partial v_{L}}{\partial z}=0 \\
& \frac{\partial w_{G}}{\partial z}=0, \quad w_{L}=0
\end{aligned}
$$

Zero gradient boundary conditions for $k$ and $\varepsilon$ were introduced at the top:

$$
\frac{\partial k}{\partial z}=\frac{\partial \varepsilon}{\partial z}=0
$$

\section{Numerical Solution Procedure}

The differential equations were integrated over staggered finite control volumes. A hybrid differencing scheme was employed to approximate the convection and diffusion terms. A fully implicit differencing scheme was applied for transient terms. The resulting systems of equations were solved using the general purpose computer program PHOENICS. ${ }^{43)}$ Time-steps in the order of milliseconds were used in the computations. The time evolution of the velocity, pressure volume fraction and tracer distribution fields were solved. At every time step, amount of tracer and liquid in the system, and liquid circulation rate were calculated to asses the accuracy of the solution. Computations were performed until the tracer concentration at every nodal location in the system attained a value within $\pm 5 \%$ of the tracer concentration of a well mixed steady state bath.

\section{Results and Discussion}

The mixing characteristics in gas injected molten metal baths are numerically studied. First, the effects of the vessel aspect ratio $\left(H_{i} / D\right)$ and then the effects of gas flow rate on the mixing are investigated.

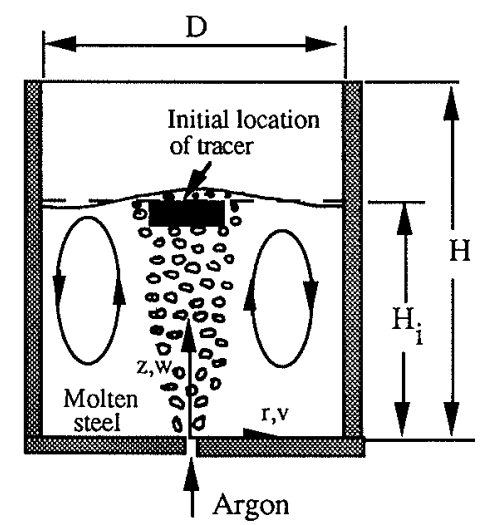

Fig. 2. Geometry, coordinate system and initial distribution of the tracer.

\subsection{Effects of the Vessel Aspect Ratio on the Mixing Rate}

A cylindrical vessel containing molten steel was considered. A tracer liquid was initially injected from the top of the bath, as seen in Fig. 2. A stream of argon was injected vertically upward through a tuyere located centrally at the bottom of the vessel. The computations were performed for four different cases. In each case, the total amount of molten steel, total amount of tracer liquid and gas injection rate were held constant. However, bath diameter and height were changed accordingly, so that the effects of the aspect ratio, $\left(H_{i} / D\right)$, on the flow structure and on the mixing in the melt could be analyzed. Preliminary computations showed that initial flow transition period is quite short (of the order of seconds). Thus, the solution of the tracer concentration field was also started from initial stationary flow conditions. The predicted mixing time can be slightly affected by this procedure. However, the trends predicted (mixing time as a function of the aspect ratio etc.) will not be affected. The initial radius of the region occupied by the tracer was one thirds of the vessel radius for each case. Its height was adjusted in such a way that the ratio of the tracer to the total liquid was the same $(0.01)$ for each case. Important parameters of the cases studied are given in Table 2. The small scale vessels are chosen so that detailed calculations of the flow field can be carried out with reasonable number of grid points. We believe that the trends predicted in our computations will apply to scaled up systems.

The grid size was varied for each case depending on the aspect ratio. The grid density, however, for each case was kept essentially same. Cell sizes in the radial direction were non-uniform. Finer grids were placed near the nozzle axis and near the side wall. It was assumed that gas temperature at the nozzle exit was the same as the melt temperature, and all fluid properties were assumed to be constant. This can be justified on the basis of the relatively small heat capacity of argon compared to molten steel.

The evolution of the flow field and tracer distribution was calculated as function of time. Typical time steps were of the order of $5 \mathrm{~ms}$. Predicted gas volume fraction contours for Cases AR1 and AR4 are given in Figs. 3(a) and $\mathbf{3 ( b )}$, respectively. Similar results (not shown) were also obtained for Cases AR2 and AR3. From these results, it is observed that around the axis of the injection nozzle, the concentration of the gas for low aspect ratios (Case AR1) is higher than that for high aspect ratios (Case AR4), but the radial extent of the two-phase gas-liquid plume is less than that of high aspect ratios (Case AR4). Notice that the liquid height in the vessel for Case AR1

Table 2. Geometry and flow parameters of the cases considered for the investigation of the effect of vessel aspect ratio on mixing.

\begin{tabular}{lccccccc}
\hline Case & $\begin{array}{c}H_{i} \\
(\mathrm{~m})\end{array}$ & $\begin{array}{c}D \\
(\mathrm{~m})\end{array}$ & $\begin{array}{c}\text { Aspect ratio } \\
H_{i} / D\end{array}$ & $\begin{array}{c}d_{o} \\
(\mathrm{~m})\end{array}$ & $\begin{array}{c}Q_{o} \\
\left(\mathrm{~m}^{2} / \mathrm{sec}\right)\end{array}$ & $\begin{array}{c}\text { Gas inlet } \\
\text { temperature }(\mathrm{K})\end{array}$ & $\begin{array}{c}\text { Grid size } \\
(r \times z)\end{array}$ \\
\hline AR1 & 0.20 & 0.40 & 0.5 & 0.002 & $3.14 \times 10^{-3}$ & 1873 & $18 \times 18$ \\
AR2 & 0.26 & 0.35 & 0.74 & 0.002 & $3.14 \times 10^{-3}$ & 1873 & $18 \times 21$ \\
AR3 & 0.355 & 0.30 & 1.18 & 0.002 & $3.14 \times 10^{-3}$ & 1873 & $15 \times 25$ \\
AR4 & 0.512 & 0.25 & 2.04 & 0.002 & $3.14 \times 10^{-3}$ & 1873 & $12 \times 23$ \\
\hline
\end{tabular}




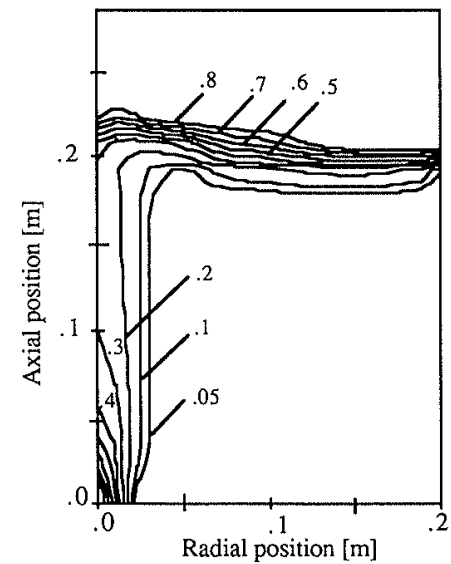

(a)

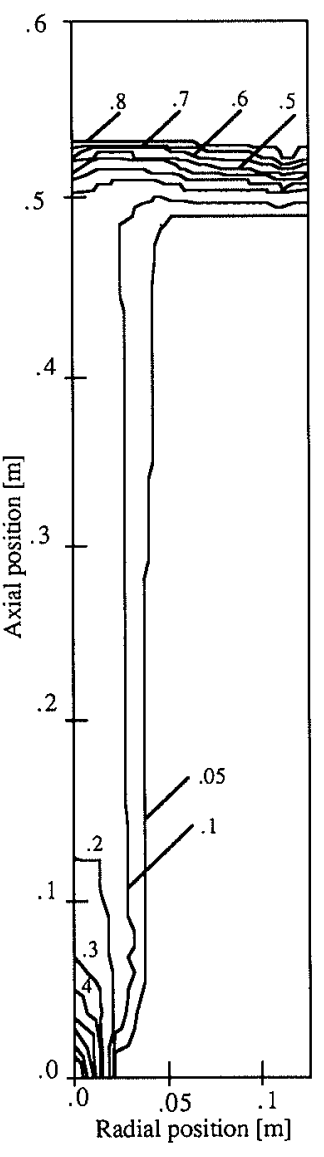

(b)
Fig. 3. Gas volume fraction contours. (a) Case AR1, (b) Case AR4.

is lower than the liquid height in the other cases, but the gas injection rates are the same. The gas concentration distribution is different owing to the differing liquid height. Its impact on mixing efficiency is explained in the following paragraphs.

The solution of the tracer concentration equation gave detailed information about the mixing in the bath. The time evolution of the tracer concentration was solved until the concentration at every nodal point in the system reached a value within $\pm 5 \%$ of the nominal well mixed tracer concentration band. At every time step, the tracer concentration was compared with final homogeneous tracer concentration. The instant at which tracer concentration at all the nodes was in the predetermined tolerance band was taken as the $95 \%$ mixing time. The liquid circulation rate was calculated using the predicted flow and phase volume fraction fields in the liquid phase.

The liquid circulation rate and the predicted $95 \%$ mixing time are plotted against the bath aspect ratio in Fig. 4. As seen, first the liquid circulation rate increases with increasing vessel aspect ratio, while mixing time decreases. Then, an opposite behavior is observed, i.e., the liquid circulation rate decreases and mixing time increases with increasing vessel aspect ratio. Thus, it is apparent that there is a maximum circulation rate and minimum mixing time corresponding to a critical vessel aspect ratio. This can be explained as follows:

When the liquid height in the bath is low, the gas

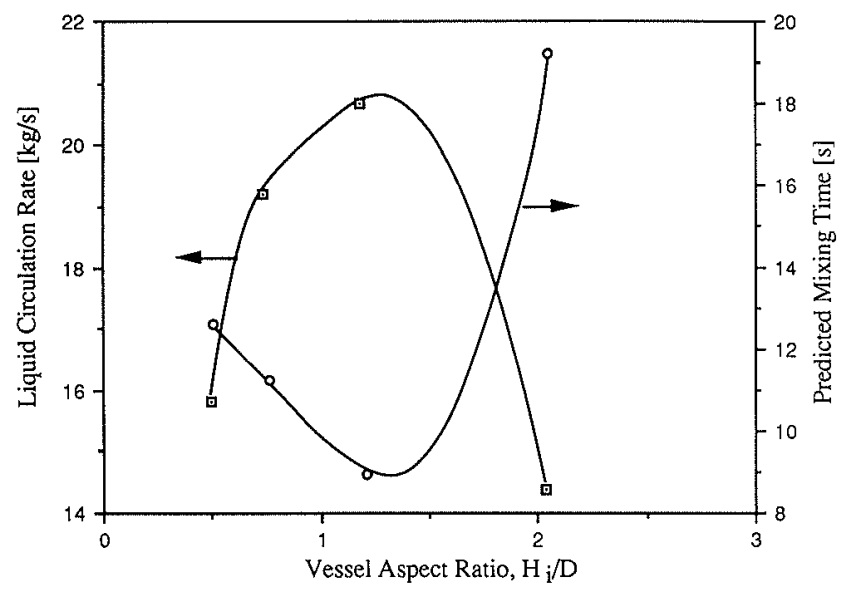

Fig. 4. Variation of the liquid circulation rate and the predicted mixing time with the vessel aspect ratio.

channels through the liquid along the injection nozzle axis. As a result, a gas column with high gas concentration forms around the axis (see Fig. 3). In such a case, effectiveness of the gas injection in inducing a motion in the liquid is less. As liquid height increases, the radial extent of the gas-liquid plume increases and hence, gas concentration within the central plume region decreases. As a result, injected gas can interact with the liquid more effectively. This leads to more liquid entrainment into the rising plume and a stronger agitation in the bath. However, with a further increase in the liquid height, the losses due to friction on the vessel walls increase. As a consequence, the liquid circulation rate may decrease and mixing time could increase.

In Fig. 4, it is also observed that the aspect ratio value of $\sim 1.5$ gives the most efficient mixing for the systems studied. Liquid circulation rate is inversely proportional to the mixing time. This indicates that bulk motion of the liquid plays an important role on the mixing. This apparent relation between the liquid circulation rate and mixing time suggest that liquid circulation rate can be used as a measure for mixing intensity in a gas injected liquid bath. However, it should be mentioned that the application of this approach to non-axisymmetric systems may not be straight forward.

The mixing time predicted by the numerical model and calculated from the correlation (Eqs. (2) and (3)) of Mazumdar and Guthrie ${ }^{37)}$ are plotted in Fig. 5. In this figure, a considerable discrepancy between the two profiles is observed. The reasons for this discrepancy could be attributed to the assumptions made in the derivation of the correlation (Eq. (2)). As mentioned earlier, Mazumdar and Guthrie assumed that the turbulent eddy viscosity and the liquid circulation velocity are uniform in the entire bath. They calculated these parameters from ad hoc relations which were also derived with additional assumptions. It should also be recalled that the constant coefficient of the correlation in Eq. (2) was determined using experimental data obtained in air-water systems. The present results appear to be realistic while the correlation by Mazumdar and Guthrie predicts very small mixing time for ladles having large aspect ratios. No optimum aspect ratio is predicted 


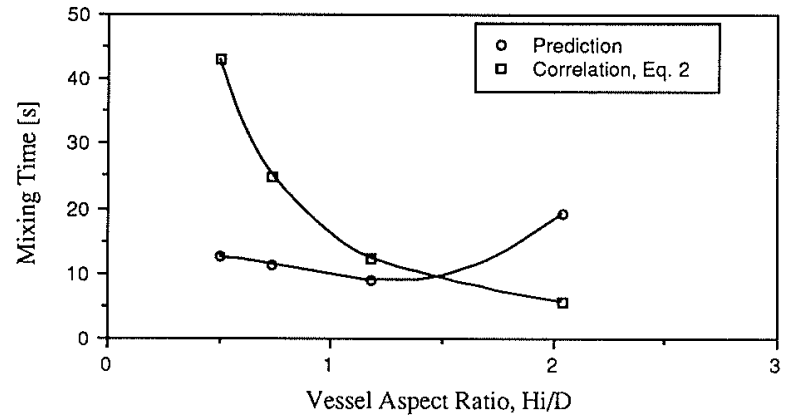

Fig. 5. Comparison of predicted mixing time with the mixing time calculated from the correlation (Eq. (2)) of Mazumdar and Guthrie. ${ }^{37}$

by the correlation. ${ }^{37)}$ It is also noted that Mazumdar and Guthrie did not indicate the ranges of parameters for which their correlation is valid.

To further investigate the mixing characteristics in gas injection systems, the specific mixing power for each case was calculated. ${ }^{44)}$ The rate at which the gas jet transfers kinetic energy to the bath is obtained as

$$
\dot{W}_{k}=\frac{1}{2} \dot{m}_{G} W_{o}^{2}
$$

where $\dot{m}_{G}$ and $W_{o}$ are gas mass flow rate and gas velocity at the nozzle exit, respectively. The energy input to the system in unit time due to the work done by the buoyancy force can be obtained from ${ }^{44)}$

$$
\dot{W}_{B}=\dot{m}_{G} R T \ln \left(1+\frac{H_{i} \rho_{L} g}{P_{a}}\right)
$$

where $R, T$ and $P_{a}$ are the gas constant, the gas temperature and the atmospheric pressure, respectively. In Eq. (21), the change in gas density due to the elevation change was taken into account. The specific mixing power, $\varepsilon_{m}$, (mixing power per unit mass of liquid) is obtained by

$$
\dot{\varepsilon}_{m}=\frac{\dot{W}_{k}+\dot{W}_{B}}{m_{L}}
$$

The specific mixing power calculated from Eq. (22) and the ratio of the gas kinetic energy (calculated from Eq. (20)) to the total work done on the liquid $\left(\dot{W}_{k}+\dot{W}_{B}\right)$ are plotted against the vessel aspect ratio in Fig. 6. As seen, mixing power increases with increasing vessel aspect ratio. The kinetic energy of the gas constitutes only a small fraction of the total work done on the liquid. This indicates that buoyancy is the dominant force for inducing motion in the bath. The contribution of gas kinetic energy at the nozzle exit is relatively high in baths with a small vessel aspect ratio.

\subsection{Effects of Gas Injection Rate on Mixing}

For evaluating the effects of gas injection rate on mixing, a cylindrical vessel of $0.3 \mathrm{~m}$ diameter was considered. The vessel contained molten steel $(1873 \mathrm{~K})$ up to $0.2 \mathrm{~m}$ from the bottom. A stream of argon was injected vertically upward through a nozzle located centrally at the bottom of the tank. The diameter of the nozzle was $2 \mathrm{~mm}$. The computations were carried out for

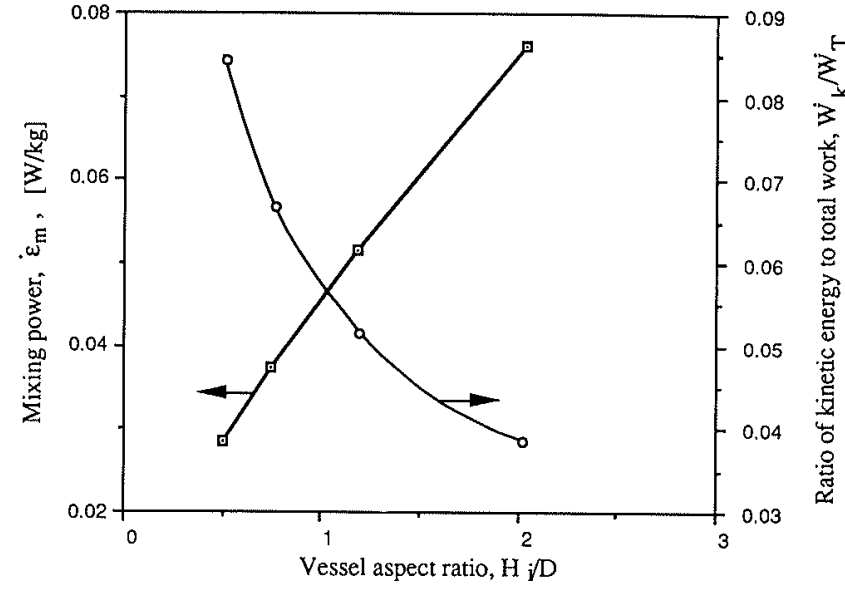

Fig. 6. Variation of specific mixing power and ratio of gas inlet kinetic energy to total work done on liquid with vessel aspect ratio.

Table 3. Gas inlet conditions.

\begin{tabular}{cccc}
\hline Case No. & $\begin{array}{c}\text { Gas inlet } \\
\text { velocity, } W_{0} \\
(\mathrm{~m} / \mathrm{sec})\end{array}$ & $\begin{array}{c}\text { Gas inlet } \\
\text { temperature } \\
(\mathrm{K})\end{array}$ & $\begin{array}{c}\text { Gas flow rate } \\
\left(\mathrm{Nm}^{3} / \mathrm{sec}\right)\end{array}$ \\
\hline IR1 & 30 & 1873 & $1.58 \times 10^{-5}$ \\
IR2 & 60 & 1873 & $3.17 \times 10^{-5}$ \\
IR3 & 90 & 1873 & $4.76 \times 10^{-5}$ \\
IR4 & 130 & 1873 & $6.84 \times 10^{-5}$ \\
IR5 & 180 & 1873 & $9.53 \times 10^{-5}$ \\
\hline
\end{tabular}

different gas injection rates, keeping all other parameters fixed. The gas inlet conditions studied are given in Table 3. The main goal of this set of computations was to investigate the effects of gas flow rate on the flow structure and on the intensity of mixing in the bath. Since it was shown earlier that the liquid circulation rate can be used as a measure for mixing efficiency, no tracer concentration equation was solved in this set of computations. Instead, the liquid circulation rate was used as a measure of mixing efficiency in the bath.

In order to investigate the grid independence of the predictions, computations were performed with different size mesh systems for Case IR3. These predictions were compared with the values that were obtained from the experimental correlations of Castillejos and Brimacombe. ${ }^{15)}$ From these comparisons, a $17 \times 22$ mesh size was chosen to be a balance between the computational cost and accuracy.

In Figs. 7(a) and 7(b), gas volume fraction maps for Cases IR 3 and IR5 are given, respectively. Similar results for Cases IR1, IR2 and IR4 (not shown) were also obtained. Radial profiles of gas volume fraction at $z=0.1 \mathrm{~m}$ are plotted in Fig. 8 for all the cases studied. In these figures, it is observed that gas concentration around the nozzle axis increases with increasing gas inlet velocity (gas flow rate). However, there is only a slight increase in radial dispersion of the gas with an increasing gas flow rate. For the cases with gas injection rate greater than $5 \times 10^{-5} \mathrm{Nm}^{3} / \mathrm{sec}$, the radial extent of the two-phase gas-liquid region is almost identical. Gas volume fraction profiles along the nozzle axis are given in Fig. 9. It is 


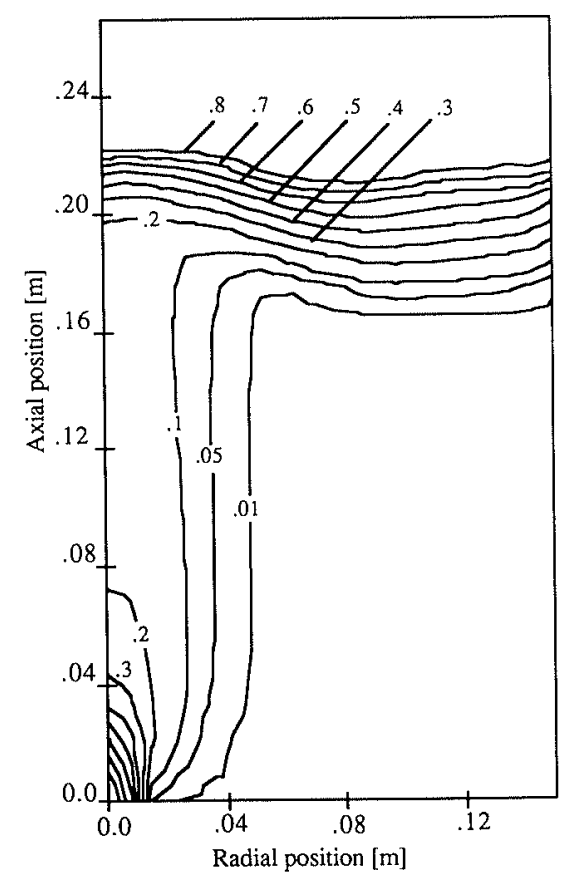

Fig. 7(a). Gas volume fraction map for Case IR3 $\left(W_{o}=90 \mathrm{~m} /\right.$ $\mathrm{sec})$.

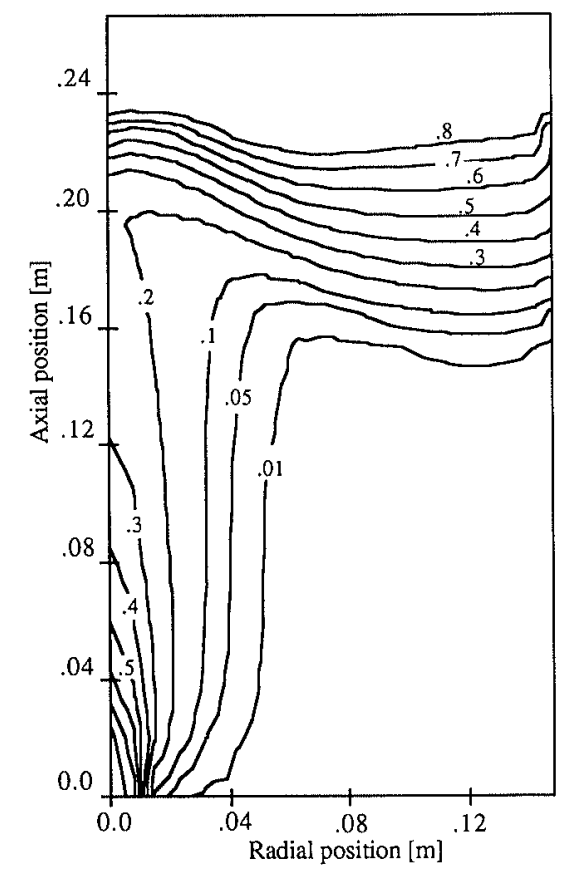

Fig. 7(b). Gas volume fraction map for Case IR5 ( $W_{o}=$ $180 \mathrm{~m} / \mathrm{sec}$ ).

also evident in this figure that gas concentration in the central region increases with increasing gas flow rate all along the axis. This means that at high gas injection rates, entrainment of the liquid into the rising plume does not increase in proportion to the gas injection rate and thus, the gas concentration increases about the axis of the injection nozzle and a gas column forms. These observations are consistent with the conclusions derived from visual studies in air-water systems by Turkoglu. ${ }^{44)}$

Superficial liquid velocity vector fields (velocity multiplied by phase volume fraction) for Cases IR3 $\left(W_{o}=90 \mathrm{~m} / \mathrm{sec}\right)$ and IR5 $\left(W_{o}=180 \mathrm{~m} / \mathrm{sec}\right)$ are given in Figs. 10(a) and 10(b), respectively. As seen, the dis-

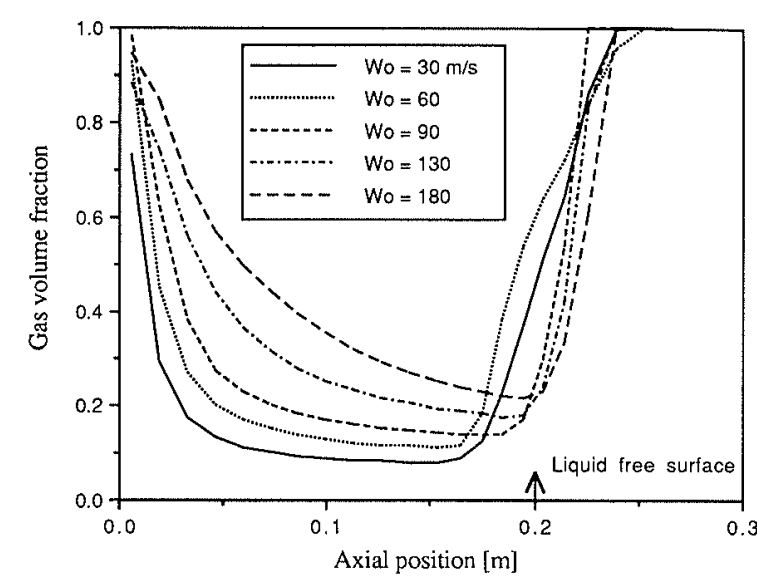

Fig. 8. Comparison of axial gas volume fraction profiles for all cases studied.

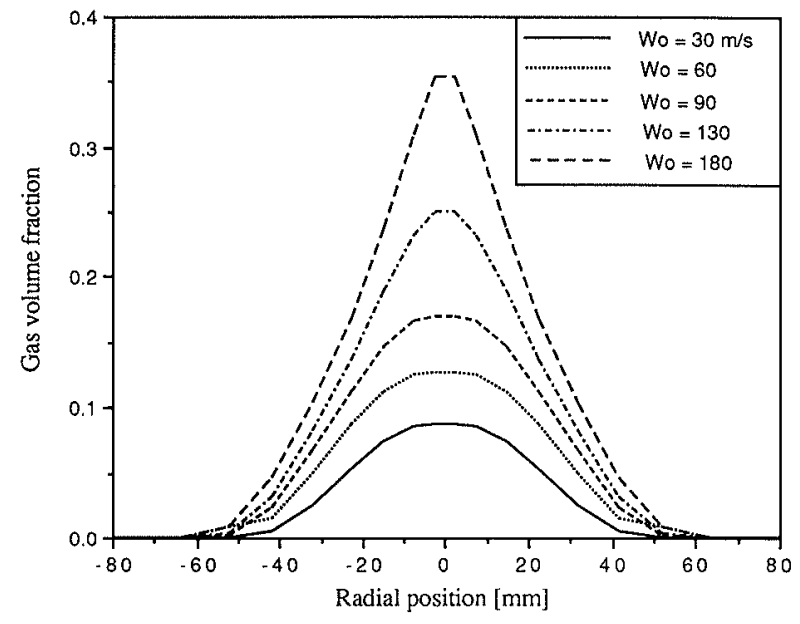

Fig. 9. Comparison of radial gas volume fraction profiles at $z=0.1 \mathrm{~m}$.

tribution of magnitude of the vectors over the flow domain is approximately the same for both cases. However, agitation in the liquid near the free surface about the injection nozzle axis is considerably higher for the high gas injection rate (Fig. 10(b)) than that for the low injection rate (Fig. 10(a)). The 'eye' of the liquid circulation is close to the bath surface for both cases. Considerable fluid motion takes place in the 'free surface' region. The velocity of the liquid is minimal at the bottom corner of the vessel. At high gas injection rates (Fig. 10(b)), the gas leaving the bath from the free surface pushes the liquid aside. In metal processing operations, this breaks up the protective slag layer covering the metal. As a result, the melt is exposed to the air. This obviously can lead to increasing oxygen absorption by the melt which negatively affects production of clean steel.

In order to quantify the intensity of the mixing in the liquid, the rate of liquid circulation was calculated for each case and compared. In Fig. 11, the circulation rates are plotted against the gas injection rate. As seen, up to a critical injection rate the circulation rate increases, but beyond this point the circulation rate remains the same. Similar results were also reported by Sahajwalla et $_{\text {al }}{ }^{45)}$ This can be explained by analyzing the gas volume fraction maps in Fig. 7 together with the liquid circulation 


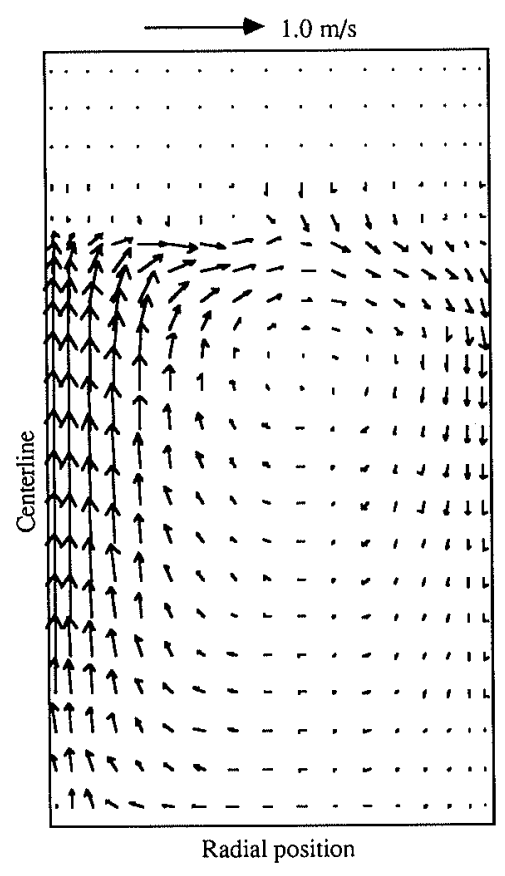

Fig. 10(a). Superficial liquid velocity vector field for Case IR3 $\left(W_{o}=90 \mathrm{~m} / \mathrm{sec}\right)$.

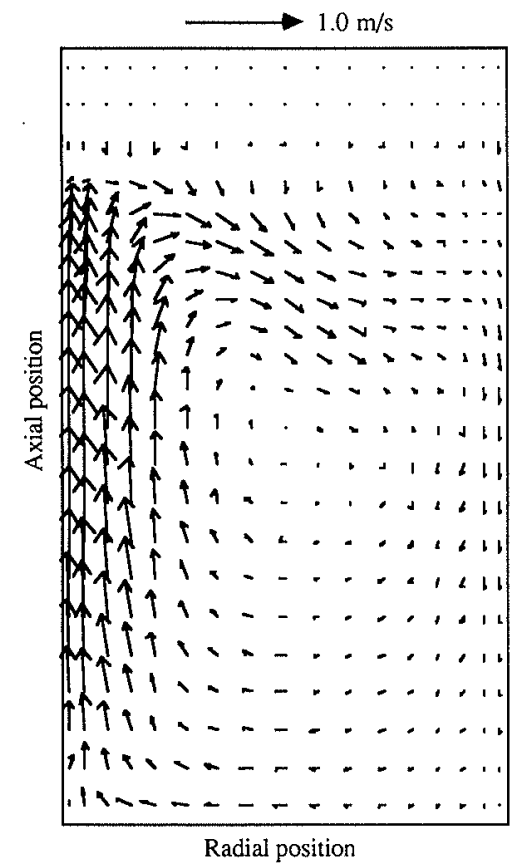

Fig. 10(b). Superficial liquid velocity vector field for Case IRS $\left(W_{o}=180 \mathrm{~m} / \mathrm{sec}\right)$.

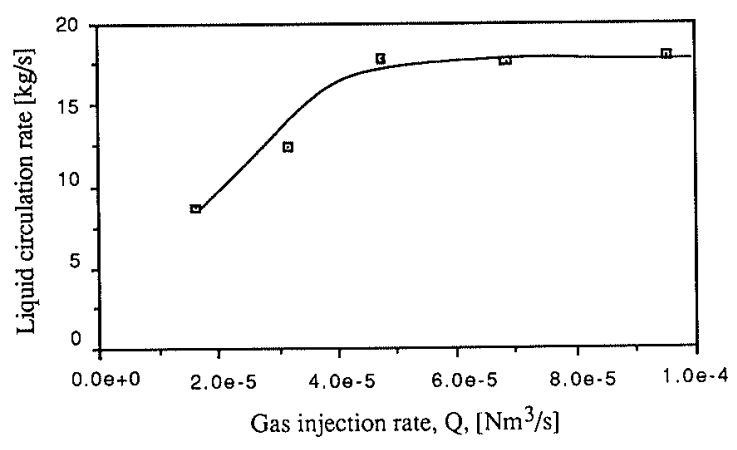

Fig. 11. Variation of the liquid circulation rate with the gas injection rate. rate profile in Fig. 11.

\section{Conclusions}

The flow structure and interaction between the injected gas and molten steel in a steelmaking ladle were analyzed numerically. The effects of bath aspect ratio and gas injection rate on mixing characteristics were investigated.

The computations revealed that the bath aspect ratio and gas flow rate play important roles on the flow processes in the bath. When the vessel aspect ratio $\left(H_{i} / D\right)$ is small (less than one), the gas can channel out of the liquid more easily and a gas column forms around the axis of the injection nozzle. In such a case, liquid entrainment into the rising gas column is due to the shearing action between the gas column and the liquid. Mixing power increases with increased bath height due to increased buoyancy work and phase dispersion. However, with increasing bath height, the losses due to friction on the walls become important. With further increase of the bath height, friction losses overweigh the mixing power gained due to increased height, and hence mixing efficiency drops. The computations revealed that a bath aspect ratio of $\sim 1.5$ gives the highest mixing efficiency. The contribution of gas kinetic energy at the nozzle exit to mixing power is very small compared to work done by the buoyancy.

In the gas-liquid two-phase plume, gas concentration increases with an increasing gas injection rate. However, the radial extent of the plume does not change significantly. At low injection rates $\left(Q<5 \times 10^{-5} \mathrm{Nm}^{3} / \mathrm{sec}\right)$, the injected gas is dispersed in the plume. With an increasing gas injection rate, a gas column forms and the mechanism of the interaction between the phases changes from bubble-liquid to gas column-liquid. A consequence of this is that after a critical gas injection rate, the mixing intensity does not increase with increasing gas flow rate. The bulk of the liquid circulates in the bath.

\section{Acknowledgment}

This study was supported in part by the Commonwealth of Pennsylvania's Ben Franklin Partnership through the Advanced Technology Center of Southeastern Pennsylvania and Air Products and Chemicals, Inc.

\section{Nomenclature}

$A_{d}$ : projected area of a bubble or droplet

$B_{d}$ : volume of a bubble or droplet

$B_{i l}$ : $\quad$-direction body force acting on phase $i$ per unit volume of the mixture

$C_{D}$ : dimensionless drag coefficient

$C_{f}$ : interphase friction coefficient

$d_{e}:$ sphere equivalent bubble/droplet diameter

$d_{0}$ : nozzle diameter

$D:$ vessel diameter

$D_{t}$ : phase dispersion coefficient, $D_{t}=v_{t} / \sigma_{d}$

$g:$ gravitational acceleration

$H_{i}$ : initial liquid height in the vessel

$H$ : height of the computational domain

I: momentum exchange between the phases per 
unit volume

$k$ : kinetic energy of turbulence

$\dot{m}_{G}$ : gas mass flow rate

$m_{L}$ : total mass of liquid in the vessel

$p:$ pressure

$Q$ : gas flow rate at normal conditions

$Q_{o}$ : volumetric gas injection rate at nozzle conditions

$r$ : radial coordinate

$\mathrm{Re}_{d}$ : bubble Reynolds number, $\mathrm{Re}_{d}=\left|V_{r}\right| d_{e} / v_{c}$

$R$ : phase volume fraction

Sc: Schmidt number

$S c_{t}:$ turbulent Schmidt number

$t:$ time

$v:$ velocity component in $r$ direction $\left(u_{e}\right)$

$\vec{V}_{r}:$ relative velocity vector

$w$ : velocity component along the $z$ axis $\left(u_{e}\right)$

$\dot{W}_{B}$ : rate of work done by the buoyancy action on gas bubbles

$\dot{W}_{k}$ : gas kinetic energy rate at the nozzle exit

$W_{o}$ : gas velocity at the nozzle exit

$z$ : axial coordinate

Greek symbols

$\varepsilon$ : dissipation rate of turbulent kinetic energy

$\dot{\varepsilon}_{m}:$ specific mixing power

$\mu_{\text {eff }}$ : effective viscosity, $\mu_{\text {eff }}=\mu+\mu_{t}$

$\mu:$ molecular viscosity

$\mu_{t}: \quad$ turbulent viscosity

$v_{t}$ : turbulent kinematic viscosity

$\Gamma_{\text {eff, } C}:$ effective mass diffusion coefficient, $\Gamma_{\text {eff, } C}=$ $\mu / S c+\mu_{t} / S c_{t}$

$\rho:$ density

$\sigma_{d}:$ dispersion Prandtl number, $\sigma_{d}=v_{t} / D_{t}$

$\tau_{m}:$ mixing time

Subscripts
$c:$ continuous phase
$d$ : dispersed phase
$G$ : gas phase
$i$ : phase indices
$j$ : phase indices
$L:$ liquid phase
$l$ : direction $(r$ or $z$ )

\section{REFERENCES}

1) J. Szekely, H. J. Wang and K. M. Kisser: Metall. Trans. B, 7B (1976), 287.

2) N. El-Kaddah and J. Szekely: Ironmaking Steelmaking, 8 (1981), 269.

3) J. H. Grevet, J. Szekely and N. El-Kaddah: Int. J. Heat Mass Transfer, 25 (1982), 487.

4) T. Deb Roy, A. K. Majumdar and D. B. Spalding: Appl. Math. Mod., 2 (1978), 146.

5) T. Deb Roy and A. K. Majumdar: J. Met., (1981), November, 42.

6) Y. Sahai and R. I. L. Guthrie: Metall. Trans. B, 13B (1982), 203.

7) Y. Sahai and R. I. L. Guthrie: Metall. Trans. B, 13B (1982).

8) D. Mazumdar, and R. I. L. Guthrie: Metall. Trans. B, 16B (1985), 83.

9) L. R. Farias and G. A. Irons: Metall. Trans. B, 17B (1984), 77.
10) G. Oryall and J. K. Brimacombe: Metall. Trans. B, 7B(1976), 391.

11) E. O. Hoefele and J. K. Brimacombe: Metall. Trans. B, 10B (1979), 631.

12) R. M. Figueria and J. Szekely: Metall. Trans. B, 16B(1985), 67.

13) A. H. Castillejos and J. K. Brimacombe: Metall. Trans, B, 18B (1987), 649.

14) A. H. Castillejos and J. K. Brimacombe: Metall. Trans. B, 18B (1987), 659.

15) A. H. Castillejos and J. K. Brimacombe: Metall. Trans. B, 20B (1989), 595.

16) J. W. McKelliget, M. Cross and R. D. Gibson: Appl. Math. Mod., 6 (1982), 469.

17) K. Y. M. Lai and M. Salcudean: Comp. Fluids, 3 (1987), 281.

18) M. Salcudean and K. Y. M. Lai: Numer. Heat Transfer, 14 (1988), 97.

19) M. Salcudean, K. Y. M. Lai and R. I. L. Guthrie: Can. J. Chem. Eng., 63 (1985), 51.

20) C. Aldham, M. Cross and N. C. Markatos: Proc. N. E. Polytechnics Mathematical Modeling and Computer Simulation Group, ed. by P. C. Hudson, (1982), 65 .

21) M. Cross and N. C. Markatos: ISS Proc. the 4th Process Technology Conf.: Mixed Gas Blowing, Vol. 4, (1984), 11.

22) M. Cross and N. C. Markatos: Society of Mining Engineers, (1984), 291.

23) P. L. T. Koh, N. C. Markatos and M. Cross: PhysicoChemical Hydrodynamics, 9 (1987), 197.

24) H. Turkoglu and B. Farouk: Metall. Trans. B, 21B (1990), 771.

25) H. Turkoglu and B. Farouk: Steelmaking Proceedings, Vol. 72 , ISS-AIME, Penn., (1989), 3.

26) H. Turkoglu and B. Farouk: Steelmaking Proceedings, Vol. 73, ISS-AIME, Penn., (1990), 377

27) S. T. Johansen and F. Boysan: Metall. Trans. B, 19B (1988), 755.

28) K. Nakanishi, T. Fujii and J. Szekely: Ironmaking Steelmaking, 3 (1975), 193.

29) U. P. Sinha and M. J. McNallan: Metall. Trans. B, 16B (1985), 850 .

30) J. Szekely, T. Lehner and C. W. Chang: Ironmaking Steelmaking, 6 (1979), 285.

31) O. Haida, T. Emi, S. Yamada and F. Sudo: Proceedings, SCAN INJECT II Conf., Lulea, Sweden, (1980), 20: 1.

32) S. Asai, T. Okamoto, J. C. He and I. Muchi: Trans. ISIJ, 23 (1983), 43.

33) M. Sano and K. Mori: Trans. Iron Steel Inst. Jpn., 23 (1983), 171.

34) D. Oymo and R. I. L. Guthrie: Proceedings, 4th Process Technology Conf., Chicago, Illinois, (1984), 45.

35) R. Matway, H. Henein, R. J. Fruehan and J. Isaacs: Proceedings, 4th Process Technology Conf., Chicago, Illinois, (1984), 39.

36) S. Joo, R. I. L. Guthrie and G. Kamal: Steelmaking Proceedings, Vol. 72, ISS-AIME, Penn., (1989), 517.

37) D. Mazumdar and R. I. L. Guthrie: Metall. Trans. B, 17B (1986), 725.

38) D. B. Spalding: Recent Advances in Numerical Methods in Fluids, ed. by C. Taylor, Pinebridge Press, London, (1980), 139.

39) A. A. Mostafa and H. C. Mongia: Int. J. Heat Mass Transfer, 30 (1987), 2583.

40) M. Ishii and N. Zuber: AIChE J., 25 (1979), 843.

41) R. Cliff, J. R. Grace and M. E. Weber: Bubbles, Drops and Particles, Academic Press Inc., New York, (1978).

42) K. H. Ng and B. D. Spalding: Physics of Fluids, 15 (1972), No. $1,20$.

43) H. I. Rosten and D. B. Spalding: Phoenics Users' Manuel, CHAM TR/100, CHAM Limited, London, (1986).

44) H. Turkoglu: Transport Processes in Gas Injected Liquid Baths, $\mathrm{Ph}$. D. Thesis, Drexel University, Philadelphia, (1990).

45) V. Sahajwalla, J. K. Brimacombe and M. Salcudean: Steelmaking Proceedings, Vol. 72, ISS-AIME, Penn., (1989), 497. 